\title{
La malaria en el mundo en 2010: ¿qué hay de nuevo acerca de esta vieja enfermedad?
}

\author{
M. Lanaspa ${ }^{\mathrm{a}, \mathrm{b}}$, M. Renom ${ }^{\mathrm{a}, \mathrm{c}}, \mathrm{Q}$. Bassat $\mathrm{a}^{\mathrm{a}, \mathrm{b}, \mathrm{d}}$ \\ ${ }^{\circ}$ Centre de Investigació en Salut Internacional de Barcelona (CRESIB). Hospital Clínic/lnstitut d'Investigacions \\ Biomèdiques August Pi i Sunyer. Universitat de Barcelona. Barcelona, España. \\ ${ }^{b}$ Centro de Investigação em Saúde de Manhiça (CISM), Manhiça. Mozambique. \\ Unidad de Salud Internacional Maternoinfantil.Hospital Universitario \\ Sant Joan de Déu-Hospital Clínic. Barcelona. España. \\ ${ }^{\circ}$ CIBER Epidemiología y Salud Pública (CIBERESP). España.
}

\section{Resumen}

El paludismo se mantiene en 2010 como uno de los grandes problemas de salud pública a nivel global. Endémico en 108 países del mundo, causa a día de hoy 250 millones de episodios clínicos y 863000 muertes anuales, principalmente en el continente africano.

El incremento en la última década de los fondos internacionales destinados a financiar actividades de control del paludismo ha condicionado un despliegue sin precedentes de herramientas de control disponibles. Así, millones de redes mosquiteras han sido distribuidas entre las poblaciones más vulnerables de África, y nuevos fármacos más potentes y eficaces han reemplazado en todos los países endémicos a los que habían quedado obsoletos. Como resultado de estos esfuerzos, y por primera vez en muchos años, estamos asistiendo a un cambio en las tendencias epidemiológicas de esta enfermedad, con una disminución de su incidencia global y, como consecuencia, una reducción de su morbimortalidad asociada. Estos progresos han espoleado a la comunidad científica a replantear de nuevo la posibilidad de erradicar esta enfermedad a nivel global. Sin embargo, y a pesar del optimismo imperante, este objetivo será imposible sin la aparición de herramientas de control nuevas y más eficaces, y no parece realista plantearlo a corto plazo. Estos esfuerzos deberán acompañarse de una agenda científica de investigación que sirva a la vez de guía para el diseño de nuevas intervenciones, pero también de mecanismo de monitorización de los progresos obtenidos. Para que el paludismo sea erradicado, también será necesario mantener un compromiso a largo plazo acompañado en paralelo de grandes esfuerzos para reforzar los frágiles sistemas de salud existentes en los países donde la enfermedad es un problema.

Este artículo intentará revisar el estado actual del paludismo, en el nuevo contexto de los esfuerzos por su eliminación global.

Palabras clave: Paludismo. Malaria. Prevención. Erradicación. Diagnóstico. Fármacos antipalúdicos. Artemisininas. Salud global. Niños.

Quique Bassat, quique.bassat@cresib.cat

Los autores declaran no presentar conflictos de intereses en relación con la preparación y publicación de este artículo. 


\section{Malaria in the world in 2010: What's new about this old scourge?}

\section{Abstract}

Malaria remains in 2010 a major global public health problem. The disease is endemic in 108 countries around the world, causing up to 250 million clinical episodes and 863,000 deaths annually, mainly in Africa.

In the last decade, the increase of international funding available to finance malaria control activities has conditioned an unprecedented uptake of the different control tools available. Thus, millions of mosquito nets have been distributed among the most vulnerable populations in Africa, and new more potent and effective drugs have replaced those that had become obsolete in most endemic countries. As a result of these efforts, and for the first time in many years, we are witnessing a change in the epidemiology of this disease, with a decrease in its overall impact, and consequently a reduction in its associated morbidity and mortality. These developments have encouraged the scientific community to reconsider the possibility of eradicating the disease globally. However, despite the prevailing optimism, this goal will be impossible without the development of new and more effective control tools, and does not seem realistic to envisage them in the short-term. These efforts must be accompanied by a scientific research agenda that will serve both as a guideline for the design of new interventions, and also as a mechanism for monitoring the progress made. If malaria is to be eradicated, long-term commitments will be necessary, in parallel with greater efforts to strengthen the fragile health systems in countries where the disease is a problem.

This article reviews the malaria situation in the light of the current efforts for its global elimination.

Key words: Malaria. Prevention and control. Eradication. Diagnosis. Antimalarials. Artemisinins. Global health. Children.

\section{El paludismo en el mundo: magnitud del problema}

Los datos recopilados por la Organización Mundial de la Salud acerca de la morbimortalidad asociada a paludismo durante el año $2009^{1}$ confirman que esta enfermedad sigue siendo uno de los principales problemas de salud pública a nivel global. Endémico en 108 países del mundo, donde cerca de 3000 millones de persones viven expuestas a infectar$\mathrm{se}^{2}$, esta enfermedad causa cerca de 250 millones de episodios clínicos y 863000 muertes anuales ${ }^{1}$. Varias iniciativas inter- nacionales han invertido esfuerzos notables en actualizar la descripción de la distribución geográfica de la enfermedad ${ }^{3-5}$. El África subsahariana es la zona que sufre sus consecuencias de manera más desproporcionada, con aproximadamente un $85 \%$ de todos los casos y hasta un $89 \%$ de todas las muertes, y en este continente siete países (República Democrática del Congo, Etiopía, Kenia, Nigeria, Sudán, Tanzania y Uganda) concentran hasta dos terceras partes de todos los ca$\operatorname{sos}^{1}$. En los países donde el paludismo es endémico, los niños pequeños y las mu- 
jeres embarazadas son los dos grupos poblacionales más vulnerables. Así, un $85 \%$ de todas las muertes se produce en la edad pediátrica ${ }^{1}$, de forma que cada 45 segundos muere un niño africano por esta enfermedad, una estadística verdaderamente intolerable.

Pero a pesar de la crudeza de estas cifras, no todo son malas noticias en lo que se refiere a esta enfermedad. En los últimos cinco años, la incidencia global de paludismo ha disminuido de forma progresiva, de forma que muchos países han visto reducidos de forma significativa no solo el número de casos, sino también las muertes atribuibles a paludismo $\mathrm{y}$, como consecuencia directa, la mortalidad infantil total. A principios del siglo XXI, se estimó que el paludismo era responsable de un $8 \%$ de las muertes en menores de cinco años a nivel global, y de hasta un $20 \%$ en el continente africano ${ }^{6}$, si bien esta cifra actualmente se ha reducido a un $16 \%$. Entre los años 2000 y 2008, una tercera parte de los países endémicos (9 en África y 29 en otros continentes) documentaron una reducción de la incidencia de paludismo superior al 50\% ${ }^{1}$, siendo estos cambios detectables incluso en países africanos con historia de alta transmisión ${ }^{8,9}$. El impacto inmediato que el descenso del paludismo conlleva en las tasas de mortalidad infantil refleja la importan- cia que supone controlar esta enfermedad de cara a cumplir los objetivos del milenio, entre los que destaca, por su ambición, el que postula una disminución en dos terceras partes de la mortalidad infantil global antes del año $2015^{10}$.

\section{Progresos en el control del paludismo}

A pesar de la naturaleza cíclica del paludismo, que podría explicar variaciones periódicas de su incidencia, parece correcto asumir que la disminución de la incidencia global y, en particular, los cambios a los que estamos asistiendo en el continente africano, se deben a la mejoría en el acceso e implementación de las herramientas de control de la enfermedad. Actualmente, las estrategias utilizadas para luchar contra el paludismo se centran en un diagnóstico precoz, el tratamiento de los episodios agudos y la prevención de nuevos casos.

Los métodos preventivos disponibles actualmente se basan en el control vectorial, e incluyen el uso de redes mosquiteras impregnadas de insecticida de larga duración, el rociamiento intradomiciliario con insecticidas y el uso de larvicidas y otras medidas medioambientales para evitar la proliferación de mosquitos en aguas estancadas.

El diagnóstico y tratamiento de los episodios agudos de forma rápida y con un 
antipalúdico altamente eficaz permite disminuir las consecuencias de la infección a nivel individual, y sirve también para evitar la posterior diseminación de la enfermedad.

Los progresos en el control de la enfermedad han empezado a observarse a medida que la financiación de estas actividades de control, dependiendo fundamentalmente de ayudas externas, se ha visto garantizada. El esfuerzo de la comunidad internacional y de las agencias financiadoras ha sido notable, y en los últimos años los fondos para la lucha contra el paludismo se han quintuplicado, pasando de 0,3 billones de dólares estadounidenses en el año 2003, a los cerca de 1,7 billones en el año 2008 1,11 . Como consecuencia del aumento de los fondos para el control del paludismo, los países han podido incrementar de forma masiva la disponibilidad de estas herramientas. Así, casi 140 millones de redes mosquiteras han sido distribuidas en países africanos en el bienio 2006-2008, logrando que hasta un $24 \%$ de todos los niños menores de cinco años del continente durmieran cada noche protegidos ${ }^{1}$. Los test diagnósticos rápidos (RDT), diseñados para suplantar al diagnóstico presuntivo clínico, también están siendo distribuidos de forma masiva, garantizando así una confirmación parasitológica antes del uso de cualquier fármaco antipalúdico. Finalmente, las combinaciones derivadas de las artemisininas, recomendadas por la OMS para el tratamiento de los episodios de malaria no complicada ${ }^{12}$, han sido ya adoptadas por la totalidad de países endémicos de África, y están siendo implementadas progresivamente, remplazando a fármacos como la cloroquina o la sulfadoxina-pirimetamina, cuya utilidad en los últimos años se había visto mermada por la aparición de resistencias por parte del parásito.

La aplicación de estrategias integradas, basadas en la combinación de todas estas herramientas de control, ha contribuido decisivamente al descenso de la incidencia del paludismo, pero estamos todavía muy lejos de poder garantizar una cobertura universal de las intervenciones en los países de mayor transmisión, tal y como se propuso alcanzar en el año 2010 en la progresión hacia el cumplimiento de los objetivos del milenio.

\section{El camino hacia la erradicación}

Los progresos observados en el control del paludismo en los últimos años han incentivado a la comunidad científica a plantearse la erradicación de esta enfermedad como una realidad que debe considerarse. Esta posibilidad, considerada tabú después del fallido experimento de la 
campaña global de erradicación liderada por la OMS en los años 50-60 del siglo pasado, ha vuelto a las agendas científicas centrando la atención pública internacional13-17. En el año 2008, la iniciativa Roll Back Malaria, apoyando un llamamiento de la Fundación Bill y Melinda Gates, declaraba que la erradicación era una obligación moral para la comunidad internacional y sugería por tanto que se convirtiese en el objetivo final de los esfuerzos. Así, los países endémicos debían trabajar para pasar de la fase de control a la fase de eliminación, y para ello proponían un plan de acción global para el paludismo (GMAP, de sus siglas en inglés) que definía los hitos a cumplirir. La transición del control a la eliminación representa un reto formidable, $y$, por encima de todo, implica un cambio de paradigma notable, puesto que ya no se trata de luchar contra la enfermedad sino que hay que eliminar hasta el último parásito para evitar la mínima posibilidad de transmisión. En la actualidad, diez países están implementando programas nacionales de eliminación, y otros seis están en la fase de preeliminación, tal y como define el GMAP. Otros nueve países (Armenia, Bahamas, Egipto, Jamaica, Marruecos, Omán, Rusia, Siria y Turkmenistán) han conseguido interrumpir la transmisión y se encuentran a un paso de la eliminación, en la fase que se conoce como prevención de la reintroducción ${ }^{1}$. Los requisitos de la OMS para certificar la eliminación del paludismo en estos países incluyen permanecer en esta fase, garantizando la no aparición de nuevos casos autóctonos durante un mínimo de cinco años. A pesar de los notables progresos descritos en algunos países en este sentido, debemos ser prudentes y realistas, asumiendo que la erradicación del paludismo no puede, en ningún caso, plantearse como un objetivo a corto plazo, ni realizarse únicamente con las herramientas de control actuales. La agenda científica deberá por tanto incluir la investigación sobre nuevos métodos diagnósticos, preventivos y de manejo clínico, proponer en paralelo estrategias innovadoras para implementarlos en aquellos países donde el paludismo se mantenga endémico y en paralelo prever medidas para reforzar los frágiles sistemas de salud existentes.

\section{El renacer de Plasmodium vivax} y la aparición de Plasmodium knowlesi

Debido a la gravedad de los cuadros causados por $P$. falciparum, y a que esta especie es responsable de la gran mayoría de las muertes por paludismo en el mundo, es comprensible que históricamente se haya producido una cierta desatención al resto de especies causantes de paludismo, y en particular a Plasmodium vivax. 
Sin embargo, en los últimos años, y coincidiendo con el cambio de paradigma anteriormente citado, el interés por $P$. vivax entre la comunidad científica ha renacido, poniéndose en entredicho algunos dogmas establecidos como el de su supuesta "benignidad"19,20. Así, una revisión de la bibliografía reciente confirma que esta especie también produce episodios graves que pueden poner en peligro la vida del paciente, a pesar de que este hecho ocurra con mucho menor frecuencia que en las infecciones por $P$. falciparum ${ }^{19-24}$, y sin haberse entendido todavía cuáles son los mecanismos fisiopatológicos ${ }^{25}$ que causan cuadros indistinguibles de los causados por $P$. falciparum. La investigación enfocada a esta especie se ha multiplicado de forma exponencial, con el objetivo de describir mejor su epidemiología y su distribución geográfica ${ }^{26}$, así como sus principales manifestaciones clínicas. P. vivax tiene la capacidad, al igual que $P$. ovale, de crear reservorios durmientes en el hígado, llamados hipnozoitos, capaces de reactivar en cualquier momento y durante periodos que pueden hacer perdurar durante años la infección inicial, reiniciando así un episodio clínico que requerirá tratamiento. Dado que para la eliminación de los hipnozoitos únicamente existe un fármaco registrado con actividad demostrada -la primaquina-, es comprensible que la lucha contra esta especie conlleve dificultades añadidas considerables. Entender la biología de los hipnozoitos, así como los condicionantes de su reactivación, y desarrollar nuevos fármacos activos o vacunas contra este estadio del ciclo vital de esta especie, son algunas de las prioridades de la investigación en el nuevo contexto de la erradicación ${ }^{27}$.

Por otro lado, en los últimos años, una especie de Plasmodium que únicamente afectaba a los primates -Plasmodium knowlesi-, ha sido añadida a las cuatro especies tradicionalmente consideradas causantes de patología en los humanos. Esta zoonosis ${ }^{28}$, detectada en zonas selváticas muy específicas de Malasia, Indonesia y Filipinas, se caracteriza por cuadros clínicos potencialmente graves, pudiendo incluso poner en peligro la vida de los pacientes ${ }^{29,30}$. Aunque los casos descritos son todavía anecdóticos, es preciso entender qué mecanismos han propiciado este salto del huésped animal al humano, y qué factores condicionan su hipotética mayor virulencia.

\section{Novedades en el diagnóstico, tratamiento y prevención}

\section{Diagnóstico}

El diagnóstico del paludismo ha sufrido 
en los últimos cinco años un cambio radical, de consecuencias muy positivas para aquellos países donde la enfermedad es más prevalente. Hasta hace poco tiempo, el método de referencia habitualmente utilizado para el diagnóstico de esta enfermedad se basaba en la visualización mediante el uso de microscopía óptica, de las formas hemáticas de Plasmodium en una muestra de sangre del paciente. Esta técnica tiene múltiples ventajas, ya que permite la identificación de la especie infectante, así como la cuantificación del grado (y por tanto de la gravedad) de la parasitación, y la evaluación de la respuesta al tratamiento al comparar muestras seriadas. Sin embargo, la microscopía es una técnica cara, metodológicamente laboriosa y que además requiere electricidad, siendo únicamente realizable por personal formado específicamente en su interpretación, motivos por los cuales su uso en la mayoría de centros de salud de países endémicos ha brillado por su ausencia. Por estos motivos, el diagnóstico de paludismo se ha basado tradicionalmente en la presencia de fiebre, principal manifestación clínica definitoria del paludismo. Durante décadas, por tanto, el manejo del paludismo ha sido empírico, basado en criterios clínicos más que en una confirmación parasitológica. Este diagnóstico y tratamiento presuntivo, altamente extendido en los países endémicos, compensaba su baja especificidad con un altísimo valor predictivo positivo, debido a la gran proporción en zonas endémicas de episodios febriles debidos a esta enfermedad ${ }^{31-33}$. En el contexto de disminución de la incidencia de los casos de paludismo en muchos países, las fiebres atribuibles a paludismo también se han visto reducidas, con lo que el diagnóstico presuntivo es cada vez más inadecua$\mathrm{do}^{34}$. Si a esto le sumamos la convicción de que el uso indiscriminado de antipalúdicos puede ser un factor determinante en la aparición de resistencias por parte del parásito $y$, por tanto, de su futura inutilización, se entiende que la OMS recomiende actualmente la confirmación parasitológica previa al uso de cualquier antipalúdico. Y hubiese sido imposible poner en práctica esta recomendación sin la aparición de los test de diagnóstico rápido (RDT), que han irrumpido en las zonas endémicas provocando una verdadera revolución diagnóstica ${ }^{35}$. Estos test basados en técnicas inmunocromatográficas de detección de antígenos del parásito en sangre, son de utilización sencilla, tienen una alta sensibilidad y especificidad y no precisan de microscopio, por lo que son ideales para la confirmación parasitológica en zonas rurales ${ }^{36,37}$. Los test 
de última generación permiten, además, la diferenciación de especie, siendo capaces de discriminar adecuadamente entre una infección por $P$. falciparum o $P$. vi$v a x^{38}$. Los grandes esfuerzos realizados para mejorar su distribución han contribuido a un aumento espectacular del número de RDT utilizados en las zonas endémicas, pasando de ser usados anecdóticamente en el año 2005, a emplearse en hasta un $22 \%$ de todos los casos de sospecha de paludismo en el continente africano en el año $2008^{12}$. Su precio, inicialmente otro problema en su implementación masiva, se ha visto reducido a niveles asequibles, siendo ahora su uso considerado coste-efectivo a la hora de decidir el manejo del paciente ${ }^{39}$.

\section{Uso de fármacos para el tratamiento y la prevención}

El paludismo es el paradigma del desequilibrio entre una altísima morbimortalidad asociada y los escasos recursos destinados a la investigación de fármacos para tratarlo ${ }^{40}$. Hasta hace pocos años, la inversión en el desarrollo de antipalúdicos era anecdótica, y se confiaba el control global de la enfermedad a un número reducido de fármacos. La aparición y rápida diseminación global de resistencias parasitarias a estos fármacos, y notablemente por parte de $P$. falciparum a la cloroquina, piedra angular del control de la enfermedad en la segunda mitad del siglo XX, condicionó una situación de vacío terapéutico con un consiguiente aumento de la mortalidad por paludismo en los países endémicos ${ }^{41}$. Como consecuencia de esto, y para apoyar el desarrollo de nuevos fármacos antipalúdicos, nació en 1999 la fundación sin ánimo de lucro Medicines for Malaria Venture (MMV), con el objetivo de reducir la carga de enfermedad causada por el paludismo en los países endémicos a través del descubrimiento, desarrollo y facilitación de nuevos fármacos antipalúdicos eficaces y asequibles ${ }^{42}$. Esto ha resultado en un renovado ímpetu en el desarrollo de nuevos fármacos, hallándose múltiples productos en diferentes fases de desarrollo. Quizás los más notables son aquellos derivados de la milenaria planta Artemisia annua, o artemisininas, ya utilizados para el tratamiento de las fiebres desde hace siglos, pero solo recientemente producidos de forma semisintética. Como ocurre de forma habitual en otras enfermedades infecciosas, donde la combinación farmacológica ha sido utilizada como única vía de escape frente al desarrollo de resistencias farmacológicas, la comunidad científica propuso el uso de terapias combinadas para el tratamiento del paludismo. La lógica de esta propues- 
ta era combinar un derivado de las artemisininas, fármacos con alta eficacia pero vida media muy corta, con otro fármaco de vida media más larga. La terapia combinada con artemisininas (ACT) protegería así a las artemisininas frente al desarrollo de resistencias ${ }^{43}$. En el año 2004, la OMS oficializó esta recomendación, anunciando que el tratamiento de elección de los casos de paludismo no complicado por $P$. falciparum debía basarse en las ACT. En la actualidad, los 45 países endémicos del continente africano ya tienen como primera línea de tratamiento a las $\mathrm{ACT}^{1}$. A pesar de estos cambios en las políticas de salud, se estima que el acceso a las ACT en África sigue siendo muy bajo, y un estudio de la OMS en 13 países africanos confirmó que menos del 15\% de los niños menores de cinco años con fiebre y sospecha de paludismo los recibieron'.

En sus nuevas recomendaciones para el tratamiento del paludismo (2010), la OMS amplía las ACT recomendadas a los siguientes cinco productos: artemetherlumefantrina $\left(A L, C\right.$ oartem $\left.{ }^{\oplus}\right)$, artesunatoamodiaquina (AS-AQ, Coarsucam ${ }^{\oplus}$ ), artesunato-mefloquina (AS-MQ), artesunato-sulfadoxina-pirimetamina (AS-SP) y dihidroartemisinina-piperaquina (DHA$P Q P, E^{2}$ urartesim ${ }^{\circledast}$ o Artekin $\left.{ }^{\circledast}\right)^{12}$. Una sexta combinación, artesunato-pyronaridina
(Pyramax ${ }^{\circledR}$ ) todavía no ha sido recomendada formalmente por la OMS, aunque diversos ensayos clínicos han mostrado resultados prometedores ${ }^{44,45}$.

Dado que las artemisininas son la última familia de fármacos que mantienen su eficacia incluso frente a parásitos multirresistentes, es altamente prioritario evitar que aparezcan resistencias y que estas se diseminen. Desgraciadamente, los primeros indicios de resistencia a estas drogas han sido ya documentados en varios países del sudeste asiático ${ }^{46,47}$. En este sentido, la OMS ha puesto en marcha una agresiva campaña de vigilancia de la aparición de resistencias y de contención de su diseminación, recomendando a su vez la prohibición de la venta de monoterapias de artemisininas, ya que su uso indiscriminado en muchos países del sudeste asiático ha sido relacionado con la posible aparición de resistencias ${ }^{1,12,48}$.

Para el tratamiento de Plasmodium vivax, la cloroquina sigue siendo el fármaco de elección en aquellos países donde no se han detectado tasas importantes de resistencia a este fármaco, aunque algunos países han introducido ya las ACT como tratamiento de elección independientemente de la especie infectante. A diferencia del tratamiento de $P$. falciparum, en estos casos es siempre necesario complementar el tra- 
tamiento con un fármaco hipnozoiticida, para evitar las recaídas secundarias a los estadios durmientes intrahepáticos. Como ya se ha mencionado anteriormente, el único fármaco disponible actualmente es la primaquina, con peligrosos efectos secundarios hemolíticos en aquellos pacientes con déficit de la enzima G6PDH. Como alternativa, se halla en avanzado estado de desarrollo la tafenoquina, con actividad similar a la primaquina pero con una acción mucho más rápida, permitiendo un tratamiento de tres días en vez de 14, aunque potencialmente responsable de problemas similares de seguridad a los causados por la primaquina ${ }^{49}$.

Las ACT en sus formulaciones parenterales han demostrado una mayor eficacia que la quinina parenteral en el tratamiento del paludismo grave, disminuyendo de forma significativa la mortalidad asociada a malaria grave tanto en adultos asiáticos ${ }^{50}$ como -más recientemente- en niños africanos ${ }^{51}$. Por este motivo, la OMS que ya recomendaba el uso de artesunato intravenoso en adultos con casos de paludismo grave está actualmente considerando la posibilidad de ampliar esta recomendación a la edad pediátrica.

Finalmente, cabe destacar que los fármacos antipalúdicos pueden usarse tam- bién en estrategias preventivas, y no solo terapéuticas. La profilaxis continuada es una estrategia logísticamente inviable en áreas endémicas, expone el fármaco utilizado al rápido desarrollo de resistencias, y condiciona invariablemente interferencias en el desarrollo de la inmunidad natural adquirida frente a esta infección, el único mecanismo protector natural frente a los riesgos de la enfermedad. Sin embargo, la administración de fármacos antipalúdicos a través de innovadores esquemas preventivos, como por ejemplo el tratamiento intermitente preventivo (IPT), consistente en la administración de varias pautas completas de tratamiento antipalúdico separadas en el tiempo, son actualmente consideradas una excelente aportación a la lucha contra la enfermedad. El aprovechamiento de los contactos rutinarios con los servicios de salud en el contexto de las visitas antenatales (embarazadas) o de vacunación (lactantes), permite una aplicabilidad a gran escala en aquellas poblaciones más vulnerables. El tratamiento preventivo en mujeres embarazadas es una recomendación implementada hace años en muchos de los países africanos con alta endemicidad para $P$. falciparum $^{52}$, y tiene repercusiones positivas tanto en la madre embarazada como en el crecimiento fetal y riesgo de supervi- 
vencia inicial del recién nacido ${ }^{53}$. La introducción en los programas de control del paludismo del tratamiento intermitente preventivo para lactantes acaba de ser recomendada por la OMS, como resultado de una alta eficacia demostrada en múltiples ensayos clínicos ${ }^{54}$, aunque todavía no se ha empezado a implementar de forma generalizada. Otras estrategias preventivas, como la administración en masa de drogas (MDA), muy utilizada en la primera campaña de erradicación, aunque posteriormente abandonada, han sido reactivadas para contribuir a la eliminación en focos donde la transmisión es intensa pero localizada. Serán necesarios estudios que eluciden cuáles son los fármacos más adecuados para esta estrategia y en qué momento deben aplicarse ${ }^{55,56}$.

\section{Desafíos futuros de la investigación}

Para que la erradicación sea posible, y en un marco temporal realista, deberemos asistir a grandes cambios en las estrategias de control, y esperar nuevas herramientas antipalúdicas altamente efectivas y fácilmente implementables. En este sentido, la aparición de una vacuna antipalúdica eficaz significaría un adelanto formidable en la lucha contra la enfermedad y su posterior eliminación. La vacuna RTS,S/AS02A, desarrollada y financiada conjuntamente por GlaxoSmithKline y la Malaria Vaccine Initiative (MVI) ha sido hasta la fecha la única candidata que ha demostrado una eficacia moderada pero mantenida en el tiempo contra la infección y la enfermedad clínica ${ }^{57-59}$, tanto en lactantes como en niños hasta cinco años de edad. Por este motivo, se está realizando actualmente un gran ensayo clínico de fase III que constituye el último paso necesario previo a su registro ante las autoridades regulatorias internacionales. El registro en los próximos años de esta primera vacuna y su posterior implementación a través de los programas ampliados de vacunación significará con seguridad un hito histórico, pero en ningún caso podrá sustituir a los métodos de control actualmente disponibles o a la necesidad de desarrollar nuevos ${ }^{60}$. Será necesario seguir investigando para desarrollar una nueva generación de vacunas con una mayor eficacia, que también protejan contra la infección de otras especies de Plasmodium, y que sean aplicables a otros grupos vulnerables como las mujeres embarazadas o los adultos sin inmunidad previa adquirida.

El camino hacia la eliminación también conllevará cambios conceptuales del enfoque de las estrategias antipalúdicas. Así, coincidiendo con la disminu- 
ción de la incidencia de la enfermedad, deberá producirse una transición desde los esfuerzos actuales enfocados predominantemente a la reducción de la morbilidad y mortalidad, hacia la introducción de estrategias diseñadas para interrumpir la transmisión de la infección, independientemente de la presencia o no de sintomatología clínica de los portadores de estos parásitos. Fárma$\cos ^{56} \mathrm{o}$ vacunas ${ }^{61}$ con un efecto específico sobre la transmisión mediada por vectores de un individuo infectado a otro serían de especial utilidad en la eliminación del paludismo.

Por último, la comunidad científica no puede olvidar el impacto que la irrupción de otras enfermedades infecciosas está teniendo sobre la epidemiología del resto de causas de mortalidad infantil a nivel global. La pandemia del VIH/SIDA, que ha afectado de forma desproporcionada al continente africano, ha condicionado en algunos casos verdaderos cambios en la epidemiología y gravedad de algunas infecciones, principalmente las bacterianas ${ }^{62}$. Aunque la interacción entre el paludismo y el VIH no ha sido todavía bien establecida ${ }^{63-65}$, existe suficiente evidencia que induce a pensar que el control de una enfermedad pueda contribuir positivamente al de la otra.

\section{Conclusiones}

Los importantes cambios vividos en los últimos años en relación a la disminución de la incidencia del paludismo en el mundo, así como a la disponibilidad y capacidad de implementación de nuevas y más eficaces herramientas de control, han condicionado una situación de optimismo desmesurado que ha generalizado la idea de que la eliminación del paludismo es posible y será una realidad a corto plazo. La comunidad científica debe ser prudente, realista y no repetir los mismos errores del pasado, que condenaron al fracaso a la primera campaña global de erradicación ${ }^{66}$. En este sentido, el esfuerzo hacia la erradicación debe ir acompañado de una agenda de investigación pormenorizada que sirva de guía para la evaluación de nuevos métodos y estrategias, y que al mismo tiempo documente y monitorice los avances obtenidos. La erradicación no será posible sin un esfuerzo generalizado a nivel global y requerirá una inversión y un compromiso a largo plazo, no solo en medidas de tratamiento y control sino en el refuerzo de los sistemas de salud sin los cuales la adecuada implementación de las medidas comentadas es casi imposible. El paludismo no conoce fronteras, y un fracaso en su control en un estado condicionaría sin duda la reaparición del pro- 
blema en los estados vecinos, por lo que estos esfuerzos deberán mantenerse 0 incluso reforzarse en cada país donde exista transmisión a pesar de que la en- fermedad deje de ser un problema de salud pública a nivel local. El mundo no se puede permitir desperdiciar esta segunda oportunidad.

\section{Bibliografía}

1. World Health Organization. The World malaria Report 2009 [consultado el 20/09/2010]. Disponible en http://www.who.int/malaria/world_ malaria_report_2009/en/index.html

2. Hay SI, Guerra CA, Tatem AJ, Noor AM, Snow RW. The global distribution and population at risk of malaria: past, present, and future. Lancet Infect Dis. 2004;4:327-36.

3. Guerra CA, Hay SI, Lucioparedes LS, Gikandi PW, Tatem AJ, Noor AM, et al. Assembling a global database of malaria parasite prevalence for the Malaria Atlas Project. Malar J. 2007;6:17.

4. Hay SI, Okiro EA, Gething PW, Patil AP, Tatem AJ, Guerra CA, et al. Estimating the global clinical burden of Plasmodium falciparum Malaria in 2007. PLoS Med. 2010;7(6):e1000290.

5. Hay SI, Sinka ME, Okara RM, Kabaria CW, Mbithi PM, Tago CC, et al. Developing global maps of the dominant anopheles vectors of human malaria. PLoS Med. 2010;7(2):e100 0209.

6. Black RE, Morris SS, Bryce J. Where and why are 10 million children dying every year? Lancet. 2003;361:2226-34.

7. Black RE, Cousens S, Johnson HL, Lawn JE, Rudan Y, Bassani DG, et al. Global, regional, and national causes of child mortality in 2008: a systematic analysis. Lancet. 2010;375:1969-87.

8. Ceesay SJ, Casals-Pascual C, Erskine J, Anya SE, Duah NO, Fulford AJC, et al. Changes in malaria indices between 1999 and 2007 in The Gam- bia: a retrospective analysis. Lancet. 2008;372: 1545-54.

9. O'Meara WP, Mangeni JN, Steketee R, Greenwood $B$. Changes in the burden of malaria in sub-Saharan Africa. Lancet Infect Dis. 2010;10: 545-55.

10. Newman RD. Malaria control beyond 2010. BMJ. 2010;340:c2714.

11. Roll Back Malaria partnership. Progress and impact series. Malaria Funding \& Resource Utilization: The First Decade of Roll Back Malaria. 2010 [consultado el 20/09/2010]. Disponible en www. rollbackmalaria.org/Progress/mpactSeries/re port1.html

12. World Health Organization. Guidelines for the treatment of malaria, 2.nd ed. (2010) [consultado el 29/06/2010]. Disponible en http://whqlibdoc.who.int/publications/2010/9789241547925 _eng.pdf

13. Greenwood BM, Fidock DA, Kyle DE, Kappe $\mathrm{S}$, Alonso $\mathrm{PL}$, Collins $\mathrm{FH}$, et al. Malaria: progress, perils, and prospects for eradication. J Clin Invest. 2008;118:1266-76.

14. Kappe SH, Vaughan AM, Boddey JA, Cowman AF. That was then but this is now: malaria research in the time of an eradication agenda. Science. 2010:328:862-6.

15. Kilama W, Ntoumi F. Malaria: a research agenda for the eradication era. Lancet. 2009;374: 1480-2.

16. Mills A, Lubell Y, Hanson K. Malaria eradication: the economic, financial and institutional challenge. Malar J. 2008;7 (Suppl 1):S11. 
17. Wells TN, Alonso PL, Gutteridge WE. New medicines to improve control and contribute to the eradication of malaria. Nat Rev Drug Discov. 2009;8:879-91.

18. Roll back malaria partnership. The Global Malaria Action Plan (GMAP), 2008 [consultado el 20/09/2010]. Disponible en www.rollbackmalaria.org/gmap

19. Rogerson SJ, Carter R. Severe vivax malaria: newly recognised or rediscovered. PLoS Med. 2008:5(6):e136.

20. Sharma A, Khanduri U. How benign is benign tertian malaria? J Vector Borne Dis. 2009;46(2):141-4.

21. Genton B, D'Acremont V, Rare L, Baea K, Reeder JC, Alpers MP, et al. Plasmodium vivax and mixed infections are associated with severe malaria in children: a prospective cohort study from Papua New Guinea. PLoS Med. 2008;5(6): e127.

22. Tjitra $E$, Anstey NM, Sugiarto $P$, Warikar $N$, Kenangalem $E$, Karyana $M$, et al. Multidrug-resistant Plasmodium vivax associated with severe and fatal malaria: a prospective study in Papua, Indonesia. PLoS Med. 2008;5(6):e128.

23. Kochar DK, Das A, Kochar SK, Saxena V, Sirohi P, Garg S, et al. Severe Plasmodium vivax malaria: a report on serial cases from Bikaner in northwestern India. Am J Trop Med Hyg. 2009;80: 194-8.

24. Price RN, Douglas NM, Anstey NM. New developments in Plasmodium vivax malaria: severe disease and the rise of chloroquine resistance. Curr Opin Infect Dis. 2009;22:430-5.

25. Anstey NM, Russell B, Yeo TW, Price RN. The pathophysiology of vivax malaria. Trends $\mathrm{Pa}$ rasitol. 2009;25:220-7.

26. Guerra CA, Howes RE, Patil AP, Gething PW, Van Boeckel TP, Temperley WH, et al. The international limits and population at risk of Plasmo- dium vivax transmission in 2009. PLoS Negl Trop Dis. 2010;4(8):e774.

27. Mueller I, Galinski MR, Baird JK, Carlton JM, Kochar DK, Alonso PL, et al. Key gaps in the knowledge of Plasmodium vivax, a neglected human malaria parasite. Lancet Infect Dis. 2009;9: 555-66.

28. Baird JK. Malaria zoonoses. Travel Med Infect Dis. 2009;7(5):269-77.

29. Cox-Singh J, Davis TM, Lee KS, Shamsul SS, Matusop A, Ratnam S, et al. Plasmodium knowlesi malaria in humans is widely distributed and potentially life threatening. Clin Infect Dis. 2008;46:16571.

30. Daneshvar C, Davis TM, Cox-Singh J, Rafa'ee MZ, Zakaria SK, Divis PC, et al. Clinical and laboratory features of human Plasmodium knowlesi infection. Clin Infect Dis. 2009;49:852-60.

31. Chandramohan D, Jaffar S, Greenwood B. Use of clinical algorithms for diagnosing malaria. Trop Med Int Health. 2002;7(1):45-52.

32. Perkins BA, Zucker JR, Otieno J, Jafari HS, Paxton L, Redd SC, et al. Evaluation of an algorithm for integrated management of childhood illness in an area of Kenya with high malaria transmission. Bull World Health Organ. 1997;75 (Suppl 1):33-42.

33. Redd SC, Kazembe PN, Luby SP, Nwanyanwu O, Hightower AW, Ziba C, et al. Clinical algorithm for treatment of Plasmodium falciparum malaria in children. Lancet. 1996;347:223-7.

34. D'Acremont V, Lengeler C, Genton B. Reduction in the proportion of fevers associated with Plasmodium falciparum parasitaemia in Africa: a systematic review. Malar J. 2010;9:240.

35. Reyburn $H$, Mbakilwa $H$, Mwangi R, Mwerinde $\mathrm{O}$, Olomi $\mathrm{R}$, Drakeley $\mathrm{C}$, et al. Rapid diagnostic tests compared with malaria microscopy for guiding outpatient treatment of febrile illness in Tanzania: randomised trial. BMJ. 2007;334:403. 
36. Wongsrichanalai $C$, Barcus MJ, Muth S, Sutamihardja A, Wernsdorfer WH. A review of malaria diagnostic tools: microscopy and rapid diagnostic test (RDT). Am J Trop Med Hyg. 2007;77 (6 Suppl):119-27.

37. Lema OE, Carter JY, Nagelkerke N, Wangai MW, Kitenge P, Gikunda SM, et al. Comparison of five methods of malaria detection in the outpatient setting. Am J Trop Med Hyg. 1999;60:177-82.

38. Meena $M$, Joshi $D$, Joshi $R$, Sridhar $S$, Waghdhare S, Gangane N, et al. Accuracy of a multispecies rapid diagnostic test kit for detection of malarial parasite at the point of care in a low endemicity region. Trans R Soc Trop Med Hyg. 2009;103:1237-44.

39. Shillcutt S, Morel C, Goodman C, Coleman P, Bell D, Whitty CJ, et al. Cost-effectiveness of malaria diagnostic methods in sub-Saharan Africa in an era of combination therapy. Bull World Health Organ. 2008;86:101-10.

40. Trouiller $P$, Olliaro $P$, Torreele E, Orbinski J, Laing R, Ford N. Drug development for neglected diseases: a deficient market and a public-health policy failure. Lancet. 2002;359:2188-94.

41. Trape JF, Pison G, Preziosi MP, Enel C, Desgrées du Loû A, Delaunay $V$, et al. Impact of chloroquine resistance on malaria mortality. C R Acad Sci III. 1998;321:689-97.

42. Medicines for Malaria Venture (MMV). Mission and vision [consultado el 20/09/2010]. Disponible en www.mmv.org/about-us

43. White NJ, Olliaro PL. Strategies for the prevention of antimalarial drug resistance: rationale for combination chemotherapy for malaria. Parasitol Today. 1996;12:399-401.

44. Ramharter M, Kurth F, Schreier AC, Nemeth J, Glasenapp I, Bélard S, et al. Fixed-dose pyronaridine-artesunate combination for treatment of uncomplicated falciparum malaria in pediatric patients in Gabon. J Infect Dis. 2008;198:911-9.
45. Tshefu AK, Gaye O, Kayentao K, Thompson $\mathrm{R}$, Bhatt KM, Sesay SS, et al. Efficacy and safety of a fixed-dose oral combination of pyronaridine-artesunate compared with artemether-lumefantrine in children and adults with uncomplicated Plasmodium falciparum malaria: a randomised non-inferiority trial. Lancet. 2010;375:1457-67.

46. Noedl H, Se Y, Schaecher K, Smith BL, Socheat $D$, Fukuda MM. Evidence of artemisinin-resistant malaria in western Cambodia. N Engl J Med. 2008;359:2619-20.

47. Dondorp AM, Nosten F, Yi P, Das D, Phyo $A P$, Tarning J, et al. Artemisinin resistance in Plasmodium falciparum malaria. N Engl J Med. 2009; 361:455-67.

48. Maude RJ, Pontavornpinyo W, Saralamba S, Aguas R, Yeung S, Dondorp AM, et al. The last man standing is the most resistant: eliminating artemisinin-resistant malaria in Cambodia. Malar J. 2009;8:31.

49. Crockett M, Kain KC. Tafenoquine: a promising new antimalarial agent. Expert Opin Investig Drugs. 2007;16:705-15.

50. Dondorp A, Nosten F, Stepniewska K, Day $\mathrm{N}$, White N. Artesunate versus quinine for treatment of severe falciparum malaria: a randomised trial. Lancet. 2005;366:717-25.

51. Dondorp AM, Fanello $\mathrm{Cl}$, Hendriksen IC, Gomes E, Seni A, Chhaganlal KD, et al. Artesunate versus quinine in the treatment of severe falciparum malaria in African children (AQUAMAT): an open-label, randomised trial. Lancet. 2010;376(9753):1647-57. (Epub 2010 Nov 7).

52. Shulman CE, Dorman EK, Cutts F, Kawuondo K, Bulmer JN, Peshu N, et al. Intermittent sulphadoxine-pyrimethamine to prevent severe anaemia secondary to malaria in pregnancy: a randomised placebo-controlled trial. Lancet. 1999;353:632-6. 
53. Menendez C, Bardaji A, Sigauque B, Sanz $S$, Aponte JJ, Mabunda $S$, et al. Malaria prevention with IPTp during pregnancy reduces neonatal mortality. PLoS One. 2010;5(2):e9438.

54. Aponte JJ, Schellenberg D, Egan A, Breckenridge A, Carneiro I, Critchley J, et al. Efficacy and safety of intermittent preventive treatment with sulfadoxine-pyrimethamine for malaria in African infants: a pooled analysis of six randomised, placebo-controlled trials. Lancet. 2009;374:1533-42.

55. Von Seidlein L, Greenwood BM. Mass administrations of antimalarial drugs. Trends Parasitol. 2003;19:452-60.

56. White NJ. The role of anti-malarial drugs in eliminating malaria. Malar J. 2008;7 (Suppl 1):S8.

57. Alonso PL, Sacarlal J, Aponte JJ, Leach A, Macete E, Milman J, et al. Efficacy of the RTS,S/ ASO2A vaccine against Plasmodium falciparum infection and disease in young African children: randomised controlled trial. Lancet. 2004;364:1411-20.

58. Bejon P, Lusingu J, Olotu A, Leach A, Lievens $M$, Vekemans J, et al. Efficacy of RTS,S/ AS01E vaccine against malaria in children 5 to 17 months of age. N Engl J Med. 2008;359:2521-32.

59. Sacarlal J, Aide P, Aponte JJ, Renom M, Leach A, Mandomando I, et al. Long-term safety and efficacy of the RTS,S/ASO2A malaria vaccine in Mozambican children. J Infect Dis. 2009;200: 329-36.

60. Greenwood B, Targett G. Do we still need a malaria vaccine? Parasite Immunol. 2009;31:582-6.

61. Stowers A, Carter R. Current developments in malaria transmission-blocking vaccines. Expert Opin Biol Ther. 2001;1:619-28.

62. Corbett EL, Steketee RW, ter Kuile FO, Latif AS, Kamali A, Hayes RJ. HIV-1/AIDS and the control of other infectious diseases in Africa. Lancet. 2002;359:2177-87.

63. Laufer MK, van Oosterhout JJ, Thesing PC, Thumba F, Zijlstra EE, Graham SM, et al. Impact of HIV-associated immunosuppression on malaria infection and disease in Malawi. J Infect Dis. 2006; 193:872-8.

64. Herrero MD, Rivas P, Rallon NI, RamirezOlivencia G, Puente S. HIV and malaria. AIDS Rev. 2007;9:88-98.

65. Reithinger R, Kamya MR, Whitty CJ, Dorsey $\mathrm{G}$, Vermund $\mathrm{SH}$. Interaction of malaria and HIV in Africa. BMJ. 2009;338:b2141.

66. Najera JA. Malaria control: achievements, problems and strategies. Parassitologia. 2001;43 (1-2):1-89. 